\title{
Psycho-Education for Anxiety Disorders in Adults: A Systematic Review of its Effectiveness
}

\author{
Fabiana Rodrigues ${ }^{1 *}$, Ana Bártolo ${ }^{1,2}$, Emelda Pacheco ${ }^{1}$, Anabela Pereira $^{1,2,3}$, Carlos F. Silva ${ }^{1,3}$ and Celso Oliveira ${ }^{4}$ \\ ${ }^{1}$ Department of Education and Psychology, University of Aveiro, Aveiro, Portugal \\ ${ }^{2}$ CINTESIS-Center for Health Technology and Services Research, Faculty of Medicine, University of Porto, Porto, Portugal \\ ${ }^{3}$ CIDTFF-Research Centre on Didactics and Technology in the Education of Trainers, University of Aveiro, Aveiro, Portugal \\ ${ }^{4}$ Filhote" Medical Clinic, Ovar, Portugal
}

\begin{abstract}
The present study aimed to provide a comprehensive review of psycho-education in adults with formally diagnosed anxiety disorders, assessing its effectiveness in reducing the severity of anxiety symptoms, psychological distress, depression and pain, and improving quality of life and satisfaction with treatment. A systematic search was conducted using a narrative approach for extraction and synthesis of the data. Searches were undertaken between April and May 2017 in the following databases: Scopus, PubMed, Web of Science and Cochrane Central Register of Controlled Trials (CENTRAL). The studies included had been published between 2000 and 2017.2804 references were identified, but the final sample of this review only considered five studies involving 490 participants. Three of the studies used a randomized design. Most of the interventions used a face-to-face format. In addition, the internet and the telephone were delivery resources used in available interventions addressed to anxiety disorders. All intervention protocols included an educational component and anxiety symptom control skills. Psycho-education improved the psychological distress, pain and quality of life of the patients with anxiety disorders and these effects remained over time. The interventions and therapists received a good evaluation. However, we emphasize the need to develop more research with sophisticated design to test the effect of psycho-educational interventions in this setting.
\end{abstract}

Keywords: Psycho-education; Psycho-educational interventions; Anxiety disorders; Adults

\section{Introduction}

Anxiety disorders include disorders that share features of excessive fear and anxiety and related behavioral disorders [1]. Fear is the emotional response to a real or perceived imminent threat, whereas anxiety is anticipation of a future threat [1]. Anxiety disorders differ from one another in the types of objects or situations that induce fear, anxiety, or avoidance behavior, and the associated cognitive ideation [1].

Psycho-education can be understood as a behavioral treatment method that consists of an explanation to patients, in a structured group or an individual program, concerning the nature of their illness, from a multidimensional viewpoint, including familial, social, biological and pharmacological perspectives. It also provides information, support and management strategies to service providers and caregivers [2,3]. There are multiple formats, such as group intervention versus individual intervention, patients versus relatives, peer-led versus professionally led, family with patient participation versus family without patient participation [4] and, more recently, face-to-face versus web-based or online [5]. Additionally, there is a growing body of evidence to support the use of alternative delivery systems, which acknowledge that not all patients require the same type, as some patients may be helped by reading self-help books [6] or using a computer program [7]. Others could benefit from a brief psycho-educational group and others may require individual psychotherapy [8]. Mental health staff can be provided with a variety of clinical tools to choose from, enabling them to effortlessly incorporate psycho-education in routine clinical practice [4].

The multifaceted nature of psycho-education and the wide range of possible applications can have beneficial effects in a diversity of outcomes (patients' relatives or families), in various mental health settings, independently of culture or the mental health care system. Therefore, its low uptake by mental health professionals cannot be ascribed to the principles, application and effectiveness of the treatment [4]. Although the role of psycho-education in anxiety disorders received scant attention in the past, nowadays it is gaining clinicians' and consumers' interest [2], but is more frequent in anxiety symptomatology.

The lack of proper information about one's illness, can cause anxiety and uncertainty [2]. Considering the negative impact of anxiety disorders on well- being and quality of life and the economic burden on society [9] it is important to provide adequate health care interventions at an early stage. Given the lack of an overview of the efficacy of psycho-education and its interventions in formally diagnosed anxiety disorders, in this review we focus on psycho-educational interventions and psycho-education for anxiety disorders. Therefore, we aim to find out the available interventions using psycho-educational approaches for anxiety disorders, their format (face-to-face or web-based/online) and effectiveness in anxiety disorders.

\section{Methods}

This systematic review aims to identify psycho-educational interventions and brief psycho-education in the $21^{\text {st }}$ century in adults diagnosed with anxiety disorders, and their effectiveness. In the present work we intend to carry out a systematic review, identifying, evaluating and summarizing the existing research, being designed to determine if

*Corresponding author: Fabiana Rodrigues, Department of Education and Psychology, University of Aveiro, Aveiro, Portugal, Tel: +351917604429; ; E-mail: rodrigues.fabiana@ua.pt

Received June 07, 2018; Accepted July 17, 2018; Published July 24, 2018

Citation: Rodrigues F, Bártolo A, Pacheco E, Pereira A, Silva CF, et al. (2018) Psycho-Education for Anxiety Disorders in Adults: A Systematic Review of its Effectiveness. J Foren Psy 3: 142. doi: 10.4172/2475-319X.1000142.

Copyright: (c) 2018 Rodrigues F, et al. This is an open-access article distributed under the terms of the Creative Commons Attribution License, which permits unrestricted use, distribution, and reproduction in any medium, provided the original author and source are credited. 
the research shows that an intervention has evidence of effectiveness, and not to know if an intervention will work in different circumstances. The methods reported here are in accordance with the Preferred Reporting Items for Systematic Reviews and Meta-Analyses (PRISMA) statement [10]. Inclusion criteria and analysis were specified and agreed upon in advance. The protocol for this systematic review was registered on PROSPERO (CRD42017064597) and is available in full on the National Institute for Health Research NIHR HTA programme website (www.nihr.ac.uk), having followed the Preferred Reporting Items for Systematic Reviews and Meta-Analyses protocols (PRISMA-P) 2015 statement [11].

\section{Literature search strategy}

We searched the following electronic databases: Scopus, PubMed, Web of Science and Cochrane Central Register of Controlled Trials (CENTRAL). The search of the key terms was performed using the OR and AND functions and database-specific filters were used where these are available. The searches were conducted between April and May 2017. The key terms used in the search were: "Psycho-educational intervention" OR "Psycho-education" AND "anxiety disorders" AND "adults". Additional searches included AND "specific phobia", "social anxiety disorder (social phobia)", "panic disorder", "agoraphobia" and "generalized anxiety disorder". The reference lists of included articles were screened manually to identify additional studies associated with the aim of the review. Due to the small sample size, the year of publication defined in the protocol was extended from 2000 to 2017. All included articles were written in English.

\section{Study selection criteria}

This systematic review includes studies involving adults (18 years or older) with anxiety disorders formally diagnosed at the time of the study. Participants could not have any comorbidity. The psychoeducational interventions and brief psycho-education focused on anxiety disorders and were conducted by healthcare professionals. We considered studies with a randomized clinical trial (RCT) and nonexperimental design. The studies included face-to-face, web-based and booklet intervention formats. All studies used self-reporting to assess psychosocial outcomes. The severity of the anxiety symptoms was defined as the primary outcome and/or other variables (e.g. quality of life, self-efficacy, satisfaction with intervention, social support, etc.) as a secondary result. Statistically significant changes were defined by a $\mathrm{p}$-value of $\mathrm{p}<.05$.

\section{Data extraction}

The selection process was conducted in accordance with the four phases of the PRISMA Statement. The initial search identified potential eligible records. Two review researchers independently identified records through database searching. Duplicate records were removed. Subsequently, references were screened considering the titles and abstracts. Based on inclusion criteria, relevant full papers were extracted, and unsuitable studies removed. Disagreement and/ or discrepancy between the raters about the eligibility of extracted full texts were resolved through discussion with a third review team member and a final list of studies for examination was produced.

For each study, information was gathered within the following categories: (i) basic demographic information such as age, sex and specific anxiety disorder; and (ii) characterization of the available interventions including sample size, setting/delivery, intervention format, comparator, duration, follow-up, measures and outcome variables. The included studies were critically assessed for their validity by two reviewers. We assessed the quality of intervention and methodology according to (i) rationale for the intervention; (ii) detail of the intervention protocol; and (iii) level of evidence (e.g. type of study).

The initial plan for assessment of the quality of the methodology with the Cochrane Checklist was revised. Since this systematic review included heterogeneous types of study, the review team performed an appraisal of quantitative studies based on the Joanna Briggs Institute (JBI) Statistics Assessment and Review Instruments (JBI-MAStARI) critical appraisal checklist for RCTs and quasi-experimental studies (non-randomized experimental studies) [12,13]. A narrative synthesis of the studies was made, given the heterogeneity of the data regarding design, type of anxiety disorder, measures used to assess outcomes and follow-up periods.

\section{Results}

The selection process can be found in the flow diagram in Figure 1. As shown, 622 articles were identified in Scopus, 216 in PubMed, 1311 in Web of Science, 655 in Cochrane Central Register of Controlled Trials (CENTRAL) and five studies were found in other sources, amounting to 2804 articles. After application of the exclusion criteria, also based on title and abstract, 2751 records were excluded and 57 articles were selected and their full texts retrieved. Of these, 52 studies were eliminated for various reasons such as duplication, diagnosis with comorbidity, non-eligible population, psycho-educational intervention not being applied or the article being written in another language.

The systematic review included RCT studies $(n=3)[2,5,14]$ and quasi-experimental studies $(n=2)[8,15]$. The baseline sample size was $\mathrm{N}=490$ and the final sample size was $\mathrm{N}=269$ participants. The average age of participants was $\mathrm{M}=37,48(\mathrm{SD}=2.45)$, although one of the studies did not report the average age [8]. Of the studies that reported participants' sex, 243 (49.5\%) were female. In four studies, the Diagnostic Manual of Mental Disorders (DSM) was used for formal diagnosis, more specifically, DSM-IV $(n=2)[2,14]$ and DSM-IV-TR (Dijk et al.).

Concerning diagnosed disorders, the samples were heterogeneous, most studies reporting a diagnosis of panic disorder with or without agoraphobia $(\mathrm{n}=3)[2,5,8]$ followed by Social Anxiety or Social Phobia $(n=2)[8,15]$ and Generalized Anxiety Disorder $(n=2)[5,8]$, and other disorders $(\mathrm{n}=1)$ [8]. The studies by Dijk [15] and Nordmo reported the average duration of the disease $(M=24 ; M=10.9$, respectively). Four of the studies were conducted in Western countries $[5,8,15]$ and two in Eastern countries $[2,14]$.

Table 1 presents the summary of the characteristics of the included studies, more specifically, of the intervention protocols. In two of the studies, the intervention was delivered by psychologists $[5,14,15]$ and in one, by nurses [8]. Other professionals were involved in delivery of the intervention, namely, psychiatrists [2] and social workers [14]. The interventions were carried out in a clinical or hospital setting, exception of the study by Dijk [15], whose intervention took place in a university, with the approach being "participants» and «teachers» instead of "patients» and «therapists».

The studies included in this review used heterogeneous measures. The self-report measure most commonly used to evaluate social anxiety was the Social Interaction Anxiety Scale (SIAS) $(n=2)[5,15]$. All studies evaluated anxious symptomatology as an intervention outcome. For this evaluation, instruments such as the Hamilton Rating Scale for Anxiety (HAM-A) and the Beck Anxiety Inventory (BAI) $(n=2)[2,14]$ 


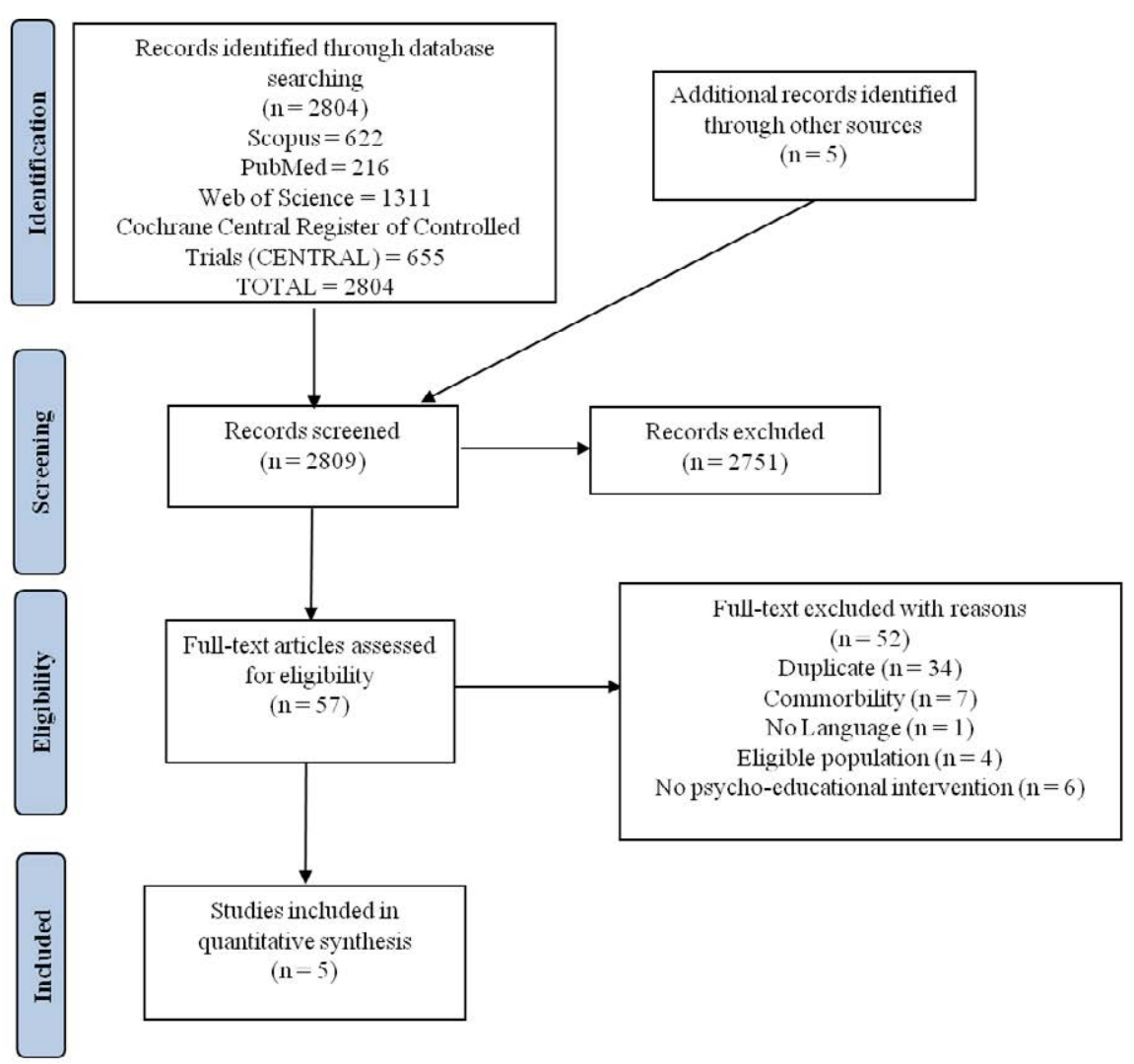

Adapted from: Moher D., Liberati, A., Tetzlaff J., Altman DG, The PRISMA Group (2009). Preferred Reporting Items for Systematic Reviews and Meta-Analysis: The PRISMA Statement. PLos Med 6(6): el000097. doi: $10.1371 /$ journal.pmed100097

Figure1: Flow diagram depicting the selection process of studies for inclusion in present systematic review.

were used, according to the specific disorder evaluated in each study, and social phobia with the Blushing subscale of the Blushing, Trembling and Sweating Questionnaire (BTSQ), Social Interaction Anxiety Scale (SIAS), Social phobia subscale of the Fear Questionnaire (FQ-SP), Social Phobia Scale (SPS) $(n=2)[5,15]$, fear with the Fear Questionnaire and Brief Fear of Negative Evaluation Scale (BFNE) $(n=2)[8,15]$, panic with the Panic Self Questionnaire (PSQ) $(n=1)$ [2] and worry with the Penn State Worry Questionnaire (PSQW) [14]. In addition, other outcomes were evaluated, namely depressive symptoms $(n=3)[2,5,14]$, quality of life $(n=1)$ [14], pain $(n=1)$ [2] and interpersonal problems $(n=1)$ [5]. Psychological distress was also evaluated $(n=1)$ [8] as well as the satisfaction with treatment $(\mathrm{n}=1)$ [8].

Regarding the theoretical rationale associated with the intervention, the study by Dijk [15] was based on the heuristic model for fear of blushing. In turn, Nordmo [5] used the Cognitive Behavioral Therapy (CBT) model for Social Anxiety Disorder (SAD), proposed by Clark and Wells [16], and Wong [14] utilized psycho-education based on CBT principles. Most studies used the face-to-face format and three of the five were group interventions $[8,14,15]$. The number of psycho-education sessions ranged from 1 to 8 . Of the five studies, the one by Dannon [2] examined the effect of a psycho-educational brochure on panic disorder. Additionally, the Self-information Booklet (SIB) was associated with psychopharmacologic treatment to test the effect of a multicomponent intervention on the frequency of panic attacks and anxiety or general functioning [2]. The results of this study suggested a reduction of pain after 1 and 3 weeks of treatment. Decreased anxiety symptoms were also found for panic attacks after 3 weeks of intervention [2]. It should also be noted that the objective of the study by Nordmo [5] was to examine the effects of a combined program involving an internet-based protocol and one face-to-face initial session of psycho-education. The results of this study showed an effect over time in anxious and depressive symptomatology. However, introduction of the psycho-education session did not lead to enhanced outcomes [5].

In general, all studies including a psycho-education condition presented an improvement of the anxiety symptoms (the main outcome), social anxiety, fears, fear of blushing, phobia, worry and distress symptoms, according to the diagnosed disorder and the assessment instruments used for the purpose. As secondary results, it was found that depressive symptoms decreased [5,14], as did pain [2], and resulted in increased quality of life related to mental health [14]. The benefits reported were obtained immediately after the intervention and were maintained at follow-up periods of 3 months, but also after 6 and 9 months. From the studies that evaluated the intervention $(n=3)$ $[5,8,15]$, participants' feedback about the therapists and interventions was positive, reinforcing the credibility of the results obtained. An interesting result is the acceptability of psycho-education in relation to comparative interventions, particularly with Mindfulness Based Cognitive Therapy (MBCT), despite non-significant differences. It is worth noting differences in the statistical approach used in the study 


\begin{tabular}{|c|c|c|c|c|c|c|c|c|}
\hline \multirow[b]{2}{*}{ Reference $^{a, b}$} & \multirow{2}{*}{\begin{tabular}{|c|} 
Sample \\
size
\end{tabular}} & \multirow[b]{2}{*}{ Setting/Delivery } & \multirow{2}{*}{$\begin{array}{l}\text { Intervention } \\
\text { format }\end{array}$} & \multirow[b]{2}{*}{ Comparator } & Duration & \multirow[b]{2}{*}{ Follow-up } & \multirow[b]{2}{*}{ Measures } & \multirow{2}{*}{$\begin{array}{c}\text { Outcome variables } \\
\text { (improvement in or reduction } \\
\text { in) }\end{array}$} \\
\hline & & & & & $\begin{array}{l}\text { (number of } \\
\text { sessions) }\end{array}$ & & & \\
\hline $\begin{array}{l}\text { Dannon et al. } \\
2002^{\mathrm{a})}\end{array}$ & $N=84$ & $\begin{array}{l}\text { Medical Center/ } \\
\text { physician or } \\
\text { psychiatrist }\end{array}$ & Paroxetine+SIB & Paroxetine & Not applicable & $\begin{array}{c}1,3 \text { and } 12 \\
\text { weeks }\end{array}$ & $\begin{array}{l}\text { HAM-A } \\
\text { HDRS } \\
\text { PSQ } \\
\text { VAS }\end{array}$ & $\begin{array}{c}\downarrow \text { scores of pain after } 1 \text { week of } \\
\text { treatment } \\
\downarrow \text { scores of anxiety, panic } \\
\text { attacks and pain after } 3 \text { weeks } \\
\text { of treatment }\end{array}$ \\
\hline $\begin{array}{l}\text { Houghton et al. } \\
2007^{\text {b) }}\end{array}$ & $N=140$ & $\begin{array}{l}\text { Outpatient mental } \\
\text { health clinic/mental } \\
\text { nurses }\end{array}$ & $\begin{array}{c}\text { Face-to-face; } \\
\text { Group intervention }\end{array}$ & None & $\begin{array}{l}4 \text { weekly } 90 \\
\text { minutes with a } 20 \\
\text { min break mid- } \\
\text { session }\end{array}$ & 12 weeks & $\begin{array}{l}\text { CORE-OM } \\
\text { Fear } \\
\text { Questionnaire } \\
\text { CSQ-8 }\end{array}$ & $\begin{array}{c}\downarrow \text { psychological distress } \\
\text { symptoms (11/44) and } \downarrow \text { phobia } \\
\text { symptoms }(6 / 55) \text { after } 3 \text { months } \\
\text { follow-up } \\
\text { Good satisfaction with } \\
\text { intervention }\end{array}$ \\
\hline $\begin{array}{l}\text { Dijk et al. } \\
2008^{b)}\end{array}$ & $\mathrm{N}=47$ & $\begin{array}{l}\text { University/ } \\
\text { Psychologists }\end{array}$ & $\begin{array}{c}\text { Face-to-face; } \\
\text { group intervention }\end{array}$ & None & $\begin{array}{c}6 \text { weekly } 2 \text {-hour } \\
\text { sessions and } \\
\text { one booster session } \\
3 \text { months after }\end{array}$ & $\begin{array}{l}3 \text { and } 12 \\
\text { months } \\
\text { (online } \\
\text { survey) }\end{array}$ & $\begin{array}{l}\text { BTSQ } \\
\text { BFNE } \\
\text { SIAS } \\
\text { FQ-SP }\end{array}$ & $\begin{array}{c}\downarrow \text { fear of blushing symptoms } \\
\downarrow \text { social anxiety complaints } \\
\text { Positive evaluation of the course } \\
\text { and the therapists }\end{array}$ \\
\hline $\begin{array}{l}\text { Nordmo et al. } \\
2015^{\text {a) }}\end{array}$ & $\mathrm{N}=37$ & $\begin{array}{l}\text { Psychological Health } \\
\text { Services and home / } \\
\text { Psychologists }\end{array}$ & $\begin{array}{l}\text { PE face-to- } \\
\text { face+GICBT } \\
\text { (online) }\end{array}$ & $\begin{array}{c}\text { GICBT } \\
\text { (without initial } \\
\text { PE session) }\end{array}$ & $\begin{array}{l}\text { PE: initial } 90 \text { min } \\
\text { session; GICBT: } \\
\text { nine modules with } \\
\text { weekly } 10 \text { min } \\
\text { telephone contact }\end{array}$ & 6 months & $\begin{array}{l}\text { SPS } \\
\text { SIAS } \\
\text { BDI } \\
\text { IIP-64 }\end{array}$ & $\begin{array}{c}\text { Effect over time regarding social } \\
\text { phobia, anxiety and depressive } \\
\text { symptoms and interpersonal } \\
\text { problems } \\
\text { Satisfaction with treatment }\end{array}$ \\
\hline $\begin{array}{l}\text { Wong et al. } \\
\qquad 2016^{\text {a) }}\end{array}$ & $N=182$ & $\begin{array}{c}\text { Clinical psychologists } \\
\text { and social worker (only } \\
\text { MBCT condition) }\end{array}$ & $\begin{array}{c}\text { face-to-face; } \\
\text { MBCT condition }\end{array}$ & $\begin{array}{l}\text { PE: face-to- } \\
\text { face; group } \\
\text { intervention } \\
\text { UC }\end{array}$ & $\begin{array}{c}\text { MBCT and PE: } \\
\text { eight weekly 2-hour } \\
\text { sessions; } \\
\text { UC: eight weeks }\end{array}$ & $\begin{array}{l}\text { PE and } \\
\text { MBCT: } 3 \text {, } \\
6 \text { and } 9 \\
\text { months; } \\
\text { UC: } 3 \\
\text { months }\end{array}$ & $\begin{array}{l}\text { BAI } \\
\text { PSWQ } \\
\text { CES-D } \\
\text { SF-12 }\end{array}$ & $\begin{array}{l}\text { Effect over time regarding } \\
\downarrow \text { anxiety and depressive } \\
\text { symptoms and } \uparrow \text { quality of life } \\
\text { related mental health and after } \\
3,6 \text { and } 9 \text { months follow-up } \\
\downarrow \text { worry symptoms after } 3 \\
\text { months follow-up } \\
\text { No significant differences } \\
\text { between PE and MBCT groups } \\
\text { Higher acceptability with PE }\end{array}$ \\
\hline
\end{tabular}

a) Randomized Clinical Trial; b) Q: Quasi-experimental study; BAI: Beck Anxiety Inventory; BDI: Beck Depression Inventory; BFNE: Brief Fear of Negative Evaluation Scale; BTSQ: Blushing subscale of the Blushing, Trembling and Sweating Questionnaire; CES-D: Center for Epidemiologic Studies Depression Scale; CORE-OM: Clinical Outcome Routine Evaluation-Outcome Measure; CSQ-8: Client Satisfaction Questionnaire; FQ-SP: Social phobia subscale of the Fear Questionnaire; GICBT: Guided Internet-delivered Cognitive Behavioural Therapy; HAM-A: Hamilton Rating Scale for Anxiety; HDRS: Hamilton Rating Depression Scale; IIP-64: Inventory of Interpersonal Problems; MBCT: Mindfulness Based Cognitive Therapy; PE: Psycho-education; PSQ: Panic Self Questionnaire; PSWQ: Penn State Worry Questionnaire; SF-12: Medical Outcomes Study Short-Form Health Survey; SIAS: Social Interaction Anxiety Scale; SIB: Self-information Booklet; SPS: Social Phobia Scale; UC: Usual Care; VAS: Visual Analog Scale; WL: Waiting List

Note: Results reported were significant.

Table 1:Descriptive summary of included studies by author and interventions used.

by Houghton [8], in which the results only reported the frequency of participants, but not detailed analysis of statistical significance.

\section{Appraisal quality}

According to the Checklist based on the Joana Briggs Appraisal of methodological quality, the studies seem to show adequate methodological rigor. All studies defined eligibility criteria and description of the intervention. The follow-up was completed (or strategies to address incomplete follow-up were utilized), the outcomes were measured reliably and similarly for treatment groups, and statistical analyses were appropriate and conclusions congruent with the data analysis.

Nevertheless, of the studies presenting the intervention, 2/5 failed to present a detailed procedure allowing replication. Only $3 / 5$ of studies contained theory or evidence-based reporting and ethics approval. All experimental studies used concealed allocation and randomization for assignment and the participants, deliverers and assessors of outcomes were blind in treatment assignment.

An important aspect of methodological quality is the internal consistency of the self-reporting measures, which was not indicated in any of the included studies. Some studies reported limitations related with a small sample, resulting in insufficient power to detect small to medium effects between groups. Similarly, despite the design used, the study by Nordmo [5] was a pilot study, limiting the statistical power of the results due to the size of the sample.

\section{Discussion}

Psycho-educational intervention appears to be a helpful intervention for a number of patients with anxiety disorders [8]. Psycho-educational intervention, normally based on cognitivebehavioral principles, showed better effects on worry symptoms and reduction of anxiety and depressive symptoms [14]. In general, the results of this systematic review suggest a clinically significant reduction of anxiety and associated symptoms, largely maintained in the followup assessment. This last result of maintenance in follow-up can be related to the fact that during the intervention or course, participants were provided with tools to cope with these symptoms, with some time being needed to practice with these before a solid effect could be expected [15]. In addition, patients with anxiety disorders can achieve a clinical and significant improvement in the symptoms of psychological distress and depression by attending such an intervention.

It is important to mention higher patient acceptance of these types of intervention [15], in spite of the low uptake by mental health professionals, which cannot be ascribed to the principles, application and effectiveness of the treatment [4]. Thus, it is important to provide mental health staff with a variety of clinical tools to choose from and enable them to effortlessly incorporate psycho-education in routine 
Citation: Rodrigues F, Bártolo A, Pacheco E, Pereira A, Silva CF, et al. (2018) Psycho-Education for Anxiety Disorders in Adults: A Systematic Review of its Effectiveness. J Foren Psy 3: 142. doi: 10.4172/2475-319X.1000142.

Page 5 of 5

clinical practice [4]. Since anxiety and uncertainty may increase due to a lack of proper information about one's illness, psycho-educational treatments might be a good option [2]. Psycho-education can become a critical ingredient in successful community care for patients with anxiety disorders and their relatives [4]. Therefore, due to its multipronged nature, psycho-education can be suited to any mental health setting irrespective of culture or the mental health care system [14].

We reinforce that this systematic served to review, identifying, evaluating and summarizing the existing literature. Thus, we search know the evidence of intervention effectiveness in a research. However, the studies show heterogeneity in their design, sample size, measurements, among others, and subgroup analyzes were not possible. Note that the existence of a possible bias since the selection criteria imply only studies published in English, reason why, works in the area that do not fulfill these conditions may be to be excluded. According to Smith [9], we considered the results of our systematic review are encouraging, suggesting that further research is needed to establish the clinical and economic effectiveness of psychotherapy for formally diagnosed anxiety disorders. Future research should investigate the efficacy of this intervention, especially in a randomized controlled trial with a robust design, with patients being randomly assigned to the psychotherapy or treatment group in the usual way (e.g. waiting list). Demographic group information should be collected about all patients, with all patients being followed up so as to minimize dropout [8].

This review emphasizes and strengthens the practice of psychoeducational interventions, namely in the diagnosis of anxiety disorders, a gap that is present in other reviews. Despite the evidence showing that web-based psycho-educational interventions are practical and effective, the nature and exact procedures of these interventions are still a matter of debate [5]. Thus, there is a need to continue to invest in assessment of the psycho-educational needs of patients with anxiety disorders, and there should be attempts to integrate other forms of non-face-to-face psychotherapy as well [5]. Future studies should develop more psychoeducational interventions at a distance, including interactive resources which have a place in health professionals' repertoire of therapeutic tools [4]. In addition, it is important that future research continues to emphasize the efficacy of these variants of psycho-education while identifying and targeting barriers to its clinical implementation [4].

We suggest additional studies with specific populations, such as firefighters and the military since people in these professions are more exposed to anxiety disorders. Although in this systematic review posttraumatic stress disorder is residual, it would be pertinent to develop studies with these populations. We recognize several limitations in this comprehensive systematic review. The characteristics of the studies included vary greatly, for example, in study design, the main focus of the intervention, the diagnostic manual used, number of sessions, delivery resources and a lack of consistency in measures and sample size. Furthermore, because we have restricted the literature search to include only quantitative studies, articles in English and published this century, relevant or less recent research published in other languages may be missing.

\section{References}

1. American Psychiatric Association. (2013) Diagnostic and Statistical Manual of Mental Disorders (DSM-5). American Psychiatric Publishing. Washington, DC,USA

2. Dannon P, lancu I, Grunhaus L (2002) Psychoeducation in Panic Disorder Patients: Effect of a Self-Information Booklet in a Randomized, masked-rater study. Depress Anxiety 16: 71-76.

3. National Institute for Health and Clinical Excellence (2006) The Management of Bipolar Affective Disorder in Adults, Children and Adolescents on Primary and Secondary Care. NICE, London

4. Economou M (2015)Psychoeducation: A Multifaceted Intervention. Int J Ment Health 44: 259-262.

5. Nordmo M, Sinding A, Carlbring P, Andersson G, Havik O, et al. (2015) Internet-Delivered Cognitive Behavioural Therapy with and without an Initial Face-to-Face Psychoeducation Session for Social Anxiety Disorder: A Pilot Randomized Controlled Trial. Internet Interventions 2: 429-436.

6. Lewis G, Anderson L, Araya R, Elgie R, Harrison G, et al. (2005) Self-Help Interventions for Mental Health Problems .Br J Gen Pract 55: 387-392.

7. Kaltenthaler E, Shakley P, Stevens K, Beverley C, Parry G, et al. (2002) A Systematic review and economic evaluation of computerised cognitive behaviour therapy for depression and anxiety. Health Technol Assess 6: 1-89.

8. Houghton S, Saxon D (2007) An Evaluation of large group CBT PsychoEducation for anxiety disorders delivered in routine practice. Patient Educ Couns 68: 107-110.

9. Smit F, Cuijpers P, Oostenbrink J, Batelaan N, de Graaf R, et al. (2006) Costs of Nine Common Mental Disorders: Implications for Curative and Preventive Psychiatry. J Ment Heal Policy Econ 9: 193-200.

10. Moher D, Liberati A, Tetzlaff J, Altman G (2009) Preferred Reporting Items for Systematic Reviews and Meta-Analyses: The PRISMA Statement. Ann Intern Med 151: 264-269.

11. Shamseer L, Moher D, Clarke M, Ghersi D, Liberati A, et al. (2015) Preferred reporting items for systematic review and meta-analysis protocols (PRISMA-P) 2015: Elaboration and Explanation. BMJ 349: 7647.

12. The Joanna Briggs Institute (JBI) 2016 a. Checklist for Quasi-Experimental Studies. Joanna Briggs Institute.

13. The Joanna Briggs Institute (JBI). 2016 b. Checklist for Randomized Controlled Trials. Australia.

14. Wong S, Yip B, Mak W, Mercer S, Cheung E, et al. (2016) Mindfulness-Based Cognitive Therapy v. Group Psychoeducation for People with Generalised Anxiety Disorder: Randomised Controlled Trial. Br J Psychiatry 209: 68-75.

15. Dijk P, Buwalda F, Jong P (2012) Dealing with fear of blushing: A Psychoeducational group intervention for fear of blushing. Clin Psychol Psychother 19: 481-487.

16. Clark D, Wells A (1995) A Cognitive Model of Social Phobia in Social Phobia Diagnosis, Assessment, and Treatment, edited by R. G. Heineberg, M. R Liebowitz, D. A. Hope, and F. R. Schneier. Guilford Press. New York 69-93 